Celgene Corporation, Lichen Teng Employee of: Celgene Corporation, Arthur Kavanaugh Grant/research support from: UCB Pharma DOI: 10.1136/annrheumdis-2019-eular.1860

\section{AB0738 DUAL NEUTRALISATION OF INTERLEUKIN (IL)-17A AND IL-17F WITH BIMEKIZUMAB IN MODERATE-TO- SEVERE PLAQUE PSORIASIS: 60-WEEK RESULTS FROM A RANDOMISED, DOUBLE-BLINDED, PHASE 2B EXTENSION STUDY}

Andrew Blauvelt ${ }^{1}$, Kim A. Papp ${ }^{2}$, Joseph F. Merola ${ }^{3}$, Alice B. Gottlieb ${ }^{4}$ Nancy Cross $^{5}$, Cynthia Madden ${ }^{5}$, Maggie Wang ${ }^{5}$, Christopher Cioffi ${ }^{6}$,Christopher E.M. Griffiths ${ }^{7} .{ }^{1}$ Oregon Medical Research Center, Portland, United States of America; ${ }^{2}$ Probity Medical Research and K Papp Clinical Research, Waterloo, Canada; ${ }^{3}$ Harvard Medical School, Brigham and Women's Hospital, Boston, United States of America; ${ }^{4}$ Icahn School of Medicine at Mount Sinai, Department of Dermatology, New York, United States of America; ${ }^{5}$ UCB Biosciences Inc., Raleigh, United States of America; ${ }^{6}$ UCB Pharma, Brussels, Belgium; ${ }^{7}$ Dermatology Centre, Salford Royal Hospital, University of Manchester, Manchester Academic Health Science Centre, Manchester, United Kingdom

Background: Psoriasis is a chronic, systemic, immune-mediated inflammatory disease associated with prominent skin manifestations. One in four patients with psoriasis also has psoriatic arthritis, ${ }^{1}$ with skin disease preceding joint manifestations in most patients. ${ }^{2}$ Interleukin (IL)-17F shares structural homology and pro-inflammatory function with IL-17A. Preclinical and early clinical data support dual neutralisation of IL-17F, together with IL-17A, as a novel targeting approach for the treatment of immune-mediated inflammatory diseases. Bimekizumab, a monoclonal antibody that potently and selectively neutralises both IL-17A and IL-17F, is in development for the treatment of psoriasis, psoriatic arthritis and ankylosing spondylitis. $^{3-5}$ In the 12-week BE ABLE 1 study (NCT02905006), bimekizumab provided rapid and substantial clinical improvements in patients with moderate-to-severe plaque psoriasis, with a safety profile consistent with previous bimekizumab studies. ${ }^{3}$

Objectives: The objectives of this Phase $2 b$ extension study (BE ABLE 2; NCT03010527) were to assess the long-term safety and efficacy of subcutaneous bimekizumab every four weeks for an additional 48 weeks (60 weeks' total exposure).

Methods: BE ABLE 1 responders $(\geq 90 \%$ reduction in Psoriasis Area Severity Index [PASI90] at Week 12) receiving placebo or bimekizumab $64 \mathrm{mg}, 160 \mathrm{mg}$, 160mg (320mg loading dose [LD]) remained on the same dose; non-responders (<PASI90 at Week 12) were re-assigned from placebo/bimekizumab $64 \mathrm{mg}$ to $160 \mathrm{mg}$ or from $160 \mathrm{mg} / 160 \mathrm{mg}$ (LD) to $320 \mathrm{mg}$ Patients previously receiving bimekizumab $320 \mathrm{mg} / 480 \mathrm{mg}$ received $320 \mathrm{mg}$ The primary variable was the exposure-adjusted incidence rate (EAIR) of treatment-emergent adverse events (TEAEs); efficacy assessments were secondary.

Results: 217 patients were enrolled. Across all doses, BE ABLE 1 responders generally maintained complete or almost complete skin clearance for up to an additional 48 weeks: PASI90: $80-100 \%$, non-responder imputation (93-100\%, observed); PASI100: $70-83 \%$ (80-96\%); Investigator's Global Assessment: $78-100 \%$ (98-100\%). PASI100 was achieved by $33-76 \%(40-82 \%)$ of non-responders (Week 48 ). EAIR of TEAEs was $206.1 / 100$ patient-years $(n=184 / 217$ [85\%]). EAIR of serious TEAEs was $6.2 / 100$ patient-years $(n=15 / 217 \quad[7 \%]) ;$ no serious TEAE was reported by $>1$ patient. The most frequent TEAEs were oral candidiasis and nasopharyngitis. No cases of suicidal ideation/behaviour, major adverse cardiac events, or inflammatory bowel disease were reported. No new safety findings were observed.

Conclusion: Nearly all BE ABLE 1 responders completing 60 weeks of bimekizumab treatment maintained complete or almost complete skin clearance, with a safety profile consistent with previous studies. ${ }^{3}$

\section{REFERENCES}

[1] Alinaghi et al. J Am Acad Dermatol 2019;80:251-65;

[2] Gladmann et al. Ann Rheum Dis 2005;64:ii14-17;

[3] Papp et al. J Am Acad Dermatol 2018;79:279-286;

[4] Van der Heijde et al. Ann Rheum Dis 2018;77:A70;

[5] Ritchlin et al. Arthritis Rheumatol 2018;70(S10):L17

Acknowledgement: The study was funded by UCB Pharma.

Disclosure of Interests: Andrew Blauvelt Grant/research support from: AbbVie, Aclaris, Akros, Allergan, Almirall, Amgen, Boehringer Ingelheim, Celgene, Dermavant, Dermira Inc, Eli Lilly and Company, Galderma,
Genentech/Roche, GlaxoSmithKline, Janssen, LEO, Meiji, Merck Sharp \& Dohme, Novartis, Pfizer, Purdue Pharma, Regeneron, Revance, Sandoz, Sanofi Genzyme, Sienna Pharmaceuticals, Sun Pharmaceuticals, UCB Pharma, Valeant, Vidac, Consultant for: AbbVie, Aclaris, Akros, Allergan, Almirall, Amgen, Boehringer Ingelheim, Celgene, Dermavant, Dermira Inc. Eli Lilly and Company, Galderma, Genentech/Roche, GlaxoSmithKline, Janssen, LEO, Meiji, Merck Sharp \& Dohme, Novartis, Pfizer, Purdue Pharma, Regeneron, Revance, Sandoz, Sanofi Genzyme, Sienna Pharmaceuticals, Sun Pharmaceuticals, UCB Pharma, Valeant, Vidac., Speakers bureau: Janssen, Regeneron, Sanofi Genzyme, Kim A. Papp Grant/ research support from: AbbVie, Akros, Allergan, Amgen, Anacor, Arcutis, Astellas, Baxalta, Boehringer Ingelheim, Bristol-Meyers Squibb, Can-Fite Biopharma, Celgene, Coherus, Dermira, Dow Pharma, Eli Lilly, Galderma, Genentech, Gilead, GlaxoSmithKline, InflaRx GmbH, Janssen, Kyowa Hakko Kirin, LEO, Medlmmune, Merck (MSD), Merck-Serono, Moberg Pharma, Novartis, Pfizer, PRCL Research, Regeneron, Roche, SanofiAventis/Genzyme, Takeda, UCB Pharma, Valeant/Bausch Health, Consultant for: AbbVie, Akros, Amgen, Arcutis, Astellas, AstraZeneca, Baxalta, Baxter, Boehringer Ingelheim, Bristol-Meyers Squibb, Can-Fite Biopharma, Celgene, Coherus, Dermira, Dow Pharma, Eli Lilly, Forward Pharma, Galderma, Genentech, Janssen, Kyowa Hakko Kirin, LEO, Meiji Seika Pharma, Merck (MSD), Merck-Serono, Mitsubishi Pharma, Novartis, Pfizer, PRCL Research, Regeneron, Roche, Sanofi-Aventis/Genzyme, Takeda UCB Pharma, Valeant/Bausch Health, Speakers bureau: AbbVie, Amgen, Astellas, Celgene, Eli Lilly, Galderma, Janssen, Kyowa Hakko Kirin, LEO, Merck (MSD), Novartis, Pfizer, Sanofi-Aventis/Genzyme, Valeant/Bausch Health, Joseph F. Merola Consultant for: Biogen IDEC, Abbvie, Amgen, Eli Lilly and Company, Novartis, Pfizer, Janssen, UCB, Samumed, Celgene, Sanofi Regeneron, Merck, and GSK, Alice B. Gottlieb Grant/ research support from: Incyte Corporation, Janssen Ortho Inc, Lilly ICOS LLC, Novartis, UCB, XBiotech, Consultant for: AbbVie, BMS, Celgene Corporation, Dermira, Incyte Corporation, Janssen Biotech, Janssen Ortho Inc, LEO Pharma, Lilly ICOS LLC, Novartis, Sun Pharmaceuticals Ltd, UCB, Speakers bureau: AbbVie, Eli Lilly and Company, Janssen Biotech, Nancy Cross Employee of: UCB Biosciences Inc, Cynthia Madden Shareholder of: UCB Biosciences Inc, Employee of: UCB Biosciences Inc, Maggie Wang Employee of: UCB Biosciences Inc, Christopher Cioff Shareholder of: UCB Biosciences Inc, Employee of: UCB Biosciences Inc, Christopher E.M. Griffiths Grant/research support from: AbbVie, Celgene, LEO, Lilly, Janssen, Novartis, Pfizer, Sandoz, UCB Pharma, Speakers bureau: AbbVie, Almirall, Bristol-Meyers Squibb, Celgene, Galderma, Janssen, Lilly, Novartis, Pfizer, Sandoz, UCB Pharma DOI: 10.1136/annrheumdis-2019-eular.4166

\section{AB0739 THE PREVALENCE OF PSYCHOLOGICAL DISORDERS IN PATIENTSWITH SEVERE PSORIASIS WITH/WITHOUT PSORIATIC ARTHRITIS IN CLINICAL PRACTICE: DATA FROM A SINGLE DERMATOLOGICAL SETTING}

Maria Chamurlieva ${ }^{1}$, Alkes Khotko ${ }^{2}$, Larisa Kruglova ${ }^{3}$, Svetlana Glukhova ${ }^{1}$, Tatiana Korotaeva ${ }^{1} .{ }^{1}$ V.A. Nasonova Research Institute of Rheumatology, Moscow, Russian Federation; ${ }^{2} \mathrm{Clinical}$ Hospital of the KKVD MH KK, Krasnodar, Russian Federation; ${ }^{3}$ Central State Medical Academy, Moscow, Russian Federation

Background: Psoriatic arthritis ( $\mathrm{PsA}$ ) and Psoriasis ( $\mathrm{PsO}$ ) are associated with different psychological disorders. Depression is a risk factor for development of PsA among PsO patients (pts). Diagnosis of PsA in dermatological practice is still problematic. The prevalence of psychological disorders in patients with severe $\mathrm{PsO}$ with/without PsA are limited in the Russian Federation.

Objectives: To study the prevalence of anxiety, depression and fatigue in pts with severe PsO with/without PsA in a single dermatological setting. Methods: 99 unselected pts (male-23/female-76) with severe plaque PsO, mean age $45.82 \pm 14.04$ years, BSA $56.35 \pm 9.11 \%$, PASI $22.50 \pm 5.13$ were included. PsA was diagnosed by the CASPAR criteria. Anxiety, depression and fatigue were identified by the Hospital Anxiety Scale (HADS-A), HADS-Depression (HADS-D) and Functional Assessment of Chronic Illness Therapy (FACIT) (points) accordingly. The HADS-A/HADS$D$ was defined as normal - 0-7; presence of disorders - 8-10; psychiatric morbidity $>11$ points accordingly. FACIT $43.6 \pm 9.4$ matched with healthy control. The lower the level of FACIT corresponded to higher fatigue. The number of pts with FACIT $\leq 30$ were calculated. All pts were treated with different synthetic DMARDs, mostly Methotrexate (subcutaneous 15$20 \mathrm{mg}$ every week), biological DMARDs according local therapy according 